\title{
A NEW APPLICATION OF ALMOST INCREASING SEQUENCES
}

\author{
BY
}

\section{H.S. ÖZARSLAN and A. KETEN}

\begin{abstract}
Bor has proved a main theorem dealing with $\left|\bar{N}, p_{n}\right|_{k}$ summability factors of infinite series. In this paper, we have generalized this theorem to the $\varphi-\left|A, p_{n}\right|_{k}$ summability factors, under weaker conditions by using an almost increasing sequence instead of a positive monotonic non-decreasing sequence.
\end{abstract}

Mathematics Subject Classification 2010: 40D25, 40F05, 40 G99.

Key words: absolute matrix summability, almost increasing sequences, infinite series.

\section{Introduction}

A positive sequence $\left(b_{n}\right)$ is said to be almost increasing if there exists a positive increasing sequence $\left(c_{n}\right)$ and two positive constants $A$ and $B$ such that $A c_{n} \leq b_{n} \leq B c_{n}$ (see [1]). Let $\sum a_{n}$ be a given infinite series with the partial sums $\left(s_{n}\right)$. Let $\left(p_{n}\right)$ be a sequence of positive numbers such that

$$
P_{n}=\sum_{v=0}^{n} p_{v} \rightarrow \infty \quad \text { as } \quad n \rightarrow \infty, \quad\left(P_{-i}=p_{-i}=0, \quad i \geq 1\right)
$$

The sequence-to-sequence transformation

$$
\sigma_{n}=\frac{1}{P_{n}} \sum_{v=0}^{n} p_{v} s_{v}
$$

defines the sequence $\left(\sigma_{n}\right)$ of the $\left(\bar{N}, p_{n}\right)$ mean of the sequence $\left(s_{n}\right)$, generated by the sequence of coefficients $\left(p_{n}\right)$ (see [5]). The series $\sum a_{n}$ is said 
to be summable $\left|\bar{N}, p_{n}\right|_{k}, k \geq 1$, if (see [2])

$$
\sum_{n=1}^{\infty}\left(\frac{P_{n}}{p_{n}}\right)^{k-1}\left|\sigma_{n}-\sigma_{n-1}\right|^{k}<\infty .
$$

Let $A=\left(a_{n v}\right)$ be a normal matrix, i.e., a lower triangular matrix of nonzero diagonal entries. Then $A$ defines the sequence-to-sequence transformation, mapping the sequence $s=\left(s_{n}\right)$ to $A s=\left(A_{n}(s)\right)$, where

$$
A_{n}(s)=\sum_{v=0}^{n} a_{n v} s_{v}, \quad n=0,1, \ldots
$$

The series $\sum a_{n}$ is said to be summable $\left|A, p_{n}\right|_{k}, k \geq 1$, if (see [7])

$$
\sum_{n=1}^{\infty}\left(\frac{P_{n}}{p_{n}}\right)^{k-1}\left|\bar{\Delta} A_{n}(s)\right|^{k}<\infty
$$

where $\bar{\Delta} A_{n}(s)=A_{n}(s)-A_{n-1}(s)$. Let $\left(\varphi_{n}\right)$ be any sequence of positive real numbers. We say that the series $\sum a_{n}$ is summable $\varphi-\left|A, p_{n}\right|_{k}, k \geq 1$, if

$$
\sum_{n=1}^{\infty} \varphi_{n}^{k-1}\left|\bar{\Delta} A_{n}(s)\right|^{k}<\infty .
$$

If we take $\varphi_{n}=\frac{P_{n}}{p_{n}}$, then $\varphi-\left|A, p_{n}\right|_{k}$ summability reduces to $\left|A, p_{n}\right|_{k}$ summability. Also, if we take $\varphi_{n}=\frac{P_{n}}{p_{n}}$ and $a_{n v}=\frac{p_{v}}{P_{n}}$, then we get $\left|\bar{N}, p_{n}\right|_{k}$ summability. Furthermore, if we take $\varphi_{n}=n$ and $a_{n v}=\frac{p_{v}}{P_{n}}$ and $p_{n}=1$ for all values of $\mathrm{n}$, then $\varphi-\left|A, p_{n}\right|_{k}$ summability reduces to $|C, 1|_{k}$ summability (see [4]).

Before stating the main theorem we must first introduce some further notations.

Given a normal matrix $A=\left(a_{n v}\right)$, we associate two lower semi matrices $\bar{A}=\left(\bar{a}_{n v}\right)$ and $\hat{A}=\left(\hat{a}_{n v}\right)$ as follows:

$$
\begin{aligned}
& \bar{a}_{n v}=\sum_{i=v}^{n} a_{n i}, \quad n, v=0,1, \ldots \\
& \hat{a}_{00}=\bar{a}_{00}=a_{00}, \quad \hat{a}_{n v}=\bar{a}_{n v}-\bar{a}_{n-1, v}, \quad n=1,2, \ldots
\end{aligned}
$$


It may be noted that $\bar{A}$ and $\hat{A}$ are the well-known matrices of series-tosequence and series-to-series transformations, respectively. Then, we have

$$
\begin{aligned}
A_{n}(s) & =\sum_{v=0}^{n} a_{n v} s_{v}=\sum_{v=0}^{n} \bar{a}_{n v} a_{v} \\
\bar{\Delta} A_{n}(s) & =\sum_{v=0}^{n} \hat{a}_{n v} a_{v} .
\end{aligned}
$$

\section{Known result}

Bor [3] has proved the following theorem for $\left|\bar{N}, p_{n}\right|_{k}$ summability method.

Theorem A. Let $\left(p_{n}\right)$ be a sequence of positive numbers such that

$$
P_{n}=O\left(n p_{n}\right) \quad \text { as } \quad n \rightarrow \infty .
$$

If $\left(X_{n}\right)$ is a positive monotonic non-decreasing sequence such that

$$
\begin{aligned}
& \lambda_{m} X_{m}=O(1) \quad \text { as } \quad m \rightarrow \infty, \\
& \sum_{n=1}^{m} n X_{n}\left|\Delta^{2} \lambda_{n}\right|=O(1), \\
& \sum_{n=1}^{m} \frac{p_{n}}{P_{n}}\left|t_{n}\right|^{k}=O\left(X_{m}\right) \text { as } m \rightarrow \infty,
\end{aligned}
$$

where $t_{n}=\frac{1}{n+1} \sum_{v=1}^{n} v a_{v}$ then the series $\sum a_{n} \lambda_{n}$ is summable $\left|\bar{N}, p_{n}\right|_{k}, k \geq 1$.

\section{The main result}

The aim of this paper is to generalize Theorem A to $\varphi-\left|A, p_{n}\right|_{k}$ summability. Now we shall prove the following theorem.

Theorem. Let $A=\left(a_{n v}\right)$ be a positive normal matrix such that

$$
\begin{aligned}
& \bar{a}_{n 0}=1, \quad n=0,1, \ldots, \\
& a_{n-1, v} \geq a_{n v}, \quad \text { for } \quad n \geq v+1, \\
& a_{n n}=O\left(\frac{p_{n}}{P_{n}}\right) \\
& \left|\hat{a}_{n, v+1}\right|=O\left(v\left|\Delta_{v} \hat{a}_{n v}\right|\right) .
\end{aligned}
$$


Let $\left(X_{n}\right)$ be an almost increasing sequence and $\left(\frac{\varphi_{n} p_{n}}{P_{n}}\right)$ be a non-increasing sequence. If conditions (11)-(13) of Theorem $A$ and

$$
\sum_{n=1}^{m} \varphi_{n}^{k-1}\left(\frac{p_{n}}{P_{n}}\right)^{k}\left|t_{n}\right|^{k}=O\left(X_{m}\right) \text { as } m \rightarrow \infty,
$$

are satisfied, then the series $\sum a_{n} \lambda_{n}$ is summable $\varphi-\left|A, p_{n}\right|_{k}, k \geq 1$.

It should be noted that if we take $\left(X_{n}\right)$ as a positive monotonic nondecreasing sequence, $a_{n v}=\frac{p_{v}}{P_{n}}$ and $\varphi_{n}=\frac{P_{n}}{p_{n}}$ in this theorem, then we get Theorem A. In this case, condition (19) reduces to condition (14) and the condition " $\left(\frac{\varphi_{n} p_{n}}{P_{n}}\right)$ is a non-increasing sequence" is automatically satisfied. We require the following lemma for the proof of the theorem.

Lemma $([6])$. Under the conditions on $\left(X_{n}\right)$ and $\left(\lambda_{n}\right)$ which are taken in the statement of our theorem, then we have the following:

$$
\begin{aligned}
& n X_{n}\left|\Delta \lambda_{n}\right|=O(1) \quad \text { as } \quad n \rightarrow \infty, \\
& \sum_{n=1}^{\infty} X_{n}\left|\Delta \lambda_{n}\right|<\infty .
\end{aligned}
$$

\section{Proof of the theorem}

Let $\left(T_{n}\right)$ denotes A-transform of the series $\sum a_{n} \lambda_{n}$. Then, by (9) and (10), we have

$$
\bar{\Delta} T_{n}=\sum_{v=1}^{n} \hat{a}_{n v} a_{v} \lambda_{v}=\sum_{v=1}^{n} \frac{\hat{a}_{n v} \lambda_{v}}{v} v a_{v} .
$$

Applying Abel's transformation to this sum, we get

$$
\begin{aligned}
\bar{\Delta} T_{n}= & \sum_{v=1}^{n-1} \Delta_{v}\left(\frac{\hat{a}_{n v} \lambda_{v}}{v}\right) \sum_{r=1}^{v} r a_{r}+\frac{\hat{a}_{n n} \lambda_{n}}{n} \sum_{r=1}^{n} r a_{r} \\
= & \frac{n+1}{n} a_{n n} \lambda_{n} t_{n}+\sum_{v=1}^{n-1} \frac{v+1}{v} \Delta_{v}\left(\hat{a}_{n v}\right) \lambda_{v} t_{v} \\
& +\sum_{v=1}^{n-1} \frac{v+1}{v} \hat{a}_{n, v+1} \Delta \lambda_{v} t_{v}+\sum_{v=1}^{n-1} \frac{1}{v} \hat{a}_{n, v+1} \lambda_{v+1} t_{v} \\
= & T_{n, 1}+T_{n, 2}+T_{n, 3}+T_{n, 4}, \quad \text { say. }
\end{aligned}
$$


To prove the theorem, by Minkowski's inequality, it is sufficient to show that $\sum_{n=1}^{\infty} \varphi_{n}^{k-1}\left|T_{n, r}\right|^{k}<\infty, \quad$ for $\quad r=1,2,3,4$. Firstly, we have that

$$
\begin{aligned}
& \sum_{n=1}^{m} \varphi_{n}^{k-1}\left|T_{n, 1}\right|^{k}=\sum_{n=1}^{m} \varphi_{n}^{k-1}\left|\frac{n+1}{n} a_{n n} \lambda_{n} t_{n}\right|^{k} \\
& =O(1) \sum_{n=1}^{m} \varphi_{n}^{k-1}\left|\lambda_{n}\right|^{k-1}\left|\lambda_{n}\right|\left|t_{n}\right|^{k}\left(\frac{p_{n}}{P_{n}}\right)^{k}=O(1) \sum_{n=1}^{m} \varphi_{n}^{k-1}\left|\lambda_{n}\right|\left|t_{n}\right|^{k}\left(\frac{p_{n}}{P_{n}}\right)^{k} \\
& =O(1) \sum_{n=1}^{m-1} \Delta\left|\lambda_{n}\right| \sum_{r=1}^{n} \varphi_{r}^{k-1}\left(\frac{p_{r}}{P_{r}}\right)^{k}\left|t_{r}\right|^{k}+O(1)\left|\lambda_{m}\right| \sum_{v=1}^{m} \varphi_{v}^{k-1}\left(\frac{p_{v}}{P_{v}}\right)^{k}\left|t_{v}\right|^{k} \\
& =O(1) \sum_{n=1}^{m-1} \Delta\left|\lambda_{n}\right| X_{n}+O(1)\left|\lambda_{m}\right| X_{m} \\
& =O(1) \sum_{n=1}^{m-1}\left|\Delta \lambda_{n}\right| X_{n}+O(1)\left|\lambda_{m}\right| X_{m}=O(1) \quad \text { as } \quad m \rightarrow \infty
\end{aligned}
$$

by virtue of the hypotheses of the theorem and lemma.

Applying Hölder's inequality with indices $k$ and $k^{\prime}$, where $k>1$ and $\frac{1}{k}+\frac{1}{k^{\prime}}=1$, as in $T_{n, 1}$, we have that

$$
\begin{aligned}
& \sum_{n=2}^{m+1} \varphi_{n}^{k-1}\left|T_{n, 2}\right|^{k}=\sum_{n=2}^{m+1} \varphi_{n}^{k-1}\left|\sum_{v=1}^{n-1} \frac{v+1}{v} \Delta_{v}\left(\hat{a}_{n v}\right) \lambda_{v} t_{v}\right|^{k} \\
& =O(1) \sum_{n=2}^{m+1} \varphi_{n}^{k-1}\left(\sum_{v=1}^{n-1}\left|\Delta_{v}\left(\hat{a}_{n v}\right)\right|\left|\lambda_{v}\right|^{k}\left|t_{v}\right|^{k}\right) \times\left(\sum_{v=1}^{n-1}\left|\Delta_{v}\left(\hat{a}_{n v}\right)\right|\right)^{k-1} \\
& =O(1) \sum_{n=2}^{m+1}\left(\frac{\varphi_{n} p_{n}}{P_{n}}\right)^{k-1}\left(\sum_{v=1}^{n-1}\left|\Delta_{v}\left(\hat{a}_{n v}\right)\right|\left|\lambda_{v}\right|^{k}\left|t_{v}\right|^{k}\right) \\
& =O(1) \sum_{v=1}^{m}\left|\lambda_{v}\right|^{k-1}\left|\lambda_{v}\right|\left|t_{v}\right|^{k} \sum_{n=v+1}^{m+1}\left(\frac{\varphi_{n} p_{n}}{P_{n}}\right)^{k-1}\left|\Delta_{v}\left(\hat{a}_{n v}\right)\right| \\
& =O(1) \sum_{v=1}^{m}\left|\lambda_{v}\right|\left|t_{v}\right|^{k}\left(\frac{\varphi_{v} p_{v}}{P_{v}}\right)^{k-1} \sum_{n=v+1}^{m+1}\left|\Delta_{v}\left(\hat{a}_{n v}\right)\right| \\
& =O(1) \sum_{v=1}^{m}\left|\lambda_{v}\right|\left|t_{v}\right|^{k} \varphi_{v}^{k-1}\left(\frac{p_{v}}{P_{v}}\right)^{k} \\
& =O(1) \quad \text { as } m \rightarrow \infty
\end{aligned}
$$


by virtue of the hypotheses of the theorem and lemma.

Now, since $v\left|\Delta \lambda_{v}\right|=O\left(\frac{1}{X_{v}}\right)=O(1)$, by (20), we have that

$$
\begin{aligned}
& \sum_{n=2}^{m+1} \varphi_{n}^{k-1}\left|T_{n, 3}\right|^{k}=\sum_{n=2}^{m+1} \varphi_{n}^{k-1}\left|\sum_{v=1}^{n-1} \frac{v+1}{v} \hat{a}_{n, v+1} \Delta \lambda_{v} t_{v}\right|^{k} \\
& =O(1) \sum_{n=2}^{m+1} \varphi_{n}^{k-1}\left(\sum_{v=1}^{n-1}\left|\hat{a}_{n, v+1}\right|\left|\Delta \lambda_{v}\right|\left|t_{v}\right|^{k}\right) \times\left(\sum_{v=1}^{n-1}\left|\hat{a}_{n, v+1}\right|\left|\Delta \lambda_{v}\right|\right)^{k-1} \\
& =O(1) \sum_{n=2}^{m+1} \varphi_{n}^{k-1}\left(\sum_{v=1}^{n-1} v\left|\Delta_{v} \hat{a}_{n v}\right|\left|\Delta \lambda_{v}\right|\left|t_{v}\right|^{k}\right) \times\left(\sum_{v=1}^{n-1} v\left|\Delta_{v} \hat{a}_{n v}\right|\left|\Delta \lambda_{v}\right|\right)^{k-1} \\
& =O(1) \sum_{n=2}^{m+1}\left(\frac{\varphi_{n} p_{n}}{P_{n}}\right)^{k-1}\left(\sum_{v=1}^{n-1} v\left|\Delta_{v} \hat{a}_{n v}\right|\left|\Delta \lambda_{v}\right|\left|t_{v}\right|^{k}\right) \\
& =O(1) \sum_{v=1}^{m} v\left|\Delta \lambda_{v}\right|\left|t_{v}\right|^{k} \sum_{n=v+1}^{m+1}\left(\frac{\varphi_{n} p_{n}}{P_{n}}\right)^{k-1}\left|\Delta_{v} \hat{a}_{n v}\right| \\
& =O(1) \sum_{v=1}^{m} v\left|\Delta \lambda_{v}\right|\left|t_{v}\right|^{k}\left(\frac{\varphi_{v} p_{v}}{P_{v}}\right)^{k-1} \sum_{n=v+1}^{m+1}\left|\Delta_{v} \hat{a}_{n v}\right| \\
& =O(1) \sum_{v=1}^{m} v\left|\Delta \lambda_{v}\right|\left|t_{v}\right|^{k} \varphi_{v}^{k-1}\left(\frac{p_{v}}{P_{v}}\right)^{k} \\
& =O(1) \sum_{v=1}^{m-1}\left|\Delta\left(v\left|\Delta \lambda_{v}\right|\right)\right| \sum_{r=1}^{v} \varphi_{r}^{k-1}\left(\frac{p_{r}}{P_{r}}\right)^{k}\left|t_{r}\right|^{k} \\
& +O(1) m\left|\Delta \lambda_{m}\right| \sum_{v=1}^{m} \varphi_{v}^{k-1}\left(\frac{p_{v}}{P_{v}}\right)^{k}\left|t_{v}\right|^{k} \\
& =O(1) \sum_{v=1}^{m-1} v X_{v}\left|\Delta^{2} \lambda_{v}\right|+O(1) \sum_{v=1}^{m-1} X_{v}\left|\Delta \lambda_{v+1}\right|+O(1) m\left|\Delta \lambda_{m}\right| X_{m} \\
& =O(1) \text { as } m \rightarrow \infty \text {, }
\end{aligned}
$$

by virtue of the hypotheses of the theorem and lemma.

Finally, using the fact $P_{n}=O\left(n p_{n}\right)$, by (11), as in $T_{n, 1}$, we have that

$$
\sum_{n=2}^{m+1} \varphi_{n}^{k-1}\left|T_{n, 4}\right|^{k}=\sum_{n=2}^{m+1} \varphi_{n}^{k-1}\left|\sum_{v=1}^{n-1} \frac{1}{v} \hat{a}_{n, v+1} \lambda_{v+1} t_{v}\right|^{k}
$$




$$
\begin{aligned}
& =O(1) \sum_{n=2}^{m+1} \varphi_{n}^{k-1}\left(\sum_{v=1}^{n-1} \frac{1}{v}\left|\hat{a}_{n, v+1}\right|\left|\lambda_{v+1}\right|^{k}\left|t_{v}\right|^{k}\right) \times\left(\sum_{v=1}^{n-1} \frac{1}{v}\left|\hat{a}_{n, v+1}\right|\right)^{k-1} \\
& =O(1) \sum_{n=2}^{m+1} \varphi_{n}^{k-1}\left(\sum_{v=1}^{n-1} \frac{1}{v}\left|\hat{a}_{n, v+1}\right|\left|\lambda_{v+1}\right|^{k}\left|t_{v}\right|^{k}\right) \times\left(\sum_{v=1}^{n-1}\left|\Delta_{v} \hat{a}_{n v}\right|\right)^{k-1} \\
& =O(1) \sum_{n=2}^{m+1}\left(\frac{\varphi_{n} p_{n}}{P_{n}}\right)^{k-1}\left(\sum_{v=1}^{n-1} \frac{1}{v}\left|\hat{a}_{n, v+1}\right|\left|\lambda_{v+1}\right|^{k}\left|t_{v}\right|^{k}\right) \\
& =O(1) \sum_{v=1}^{m} \frac{1}{v}\left|\lambda_{v+1}\right|^{k-1}\left|\lambda_{v+1}\right|\left|t_{v}\right|^{k} \sum_{n=v+1}^{m+1}\left(\frac{\varphi_{n} p_{n}}{P_{n}}\right)^{k-1}\left|\hat{a}_{n, v+1}\right| \\
& =O(1) \sum_{v=1}^{m} \frac{1}{v}\left|\lambda_{v+1}\right|\left|t_{v}\right|^{k}\left(\frac{\varphi_{v} p_{v}}{P_{v}}\right)^{k-1} \sum_{n=v+1}^{m+1}\left|\hat{a}_{n, v+1}\right| \\
& =O(1) \sum_{v=1}^{m} \frac{1}{v}\left|\lambda_{v+1}\right|\left|t_{v}\right|^{k}\left(\frac{\varphi_{v} p_{v}}{P_{v}}\right)^{k-1} \\
& =O(1) \sum_{v=1}^{m}\left|\lambda_{v+1}\right|\left|t_{v}\right|^{k} \varphi_{v}^{k-1}\left(\frac{P_{v}}{v p_{v}}\right)\left(\frac{p_{v}}{P_{v}}\right)^{k} \\
& =O(1) \sum_{v=1}^{m}\left|\lambda_{v+1}\right|\left|t_{v}\right|^{k} \varphi_{v}^{k-1}\left(\frac{p_{v}}{P_{v}}\right)^{k} \\
& =O(1) \text { as } m \rightarrow \infty,
\end{aligned}
$$

by virtue of the hypotheses of the theorem and lemma. This completes the proof of the theorem.

Corollary 1. If we take $\varphi_{n}=\frac{P_{n}}{p_{n}}$, then we get a result concerning the $\left|A, p_{n}\right|_{k}$ summability.

Corollary 2. If we take $a_{n v}=\frac{p_{v}}{P_{n}}$, then we have another a result dealing with $\left|\bar{N}, p_{n}, \varphi_{n}\right|_{k}$ summability.

Corollary 3. If we take $a_{n v}=\frac{p_{v}}{P_{n}}$ and $p_{n}=1$ for all values of $n$, then we get a result dealing with $\left|C, 1, \varphi_{n}\right|_{k}$ summability.

Corollary 4. If we take $\varphi_{n}=n, a_{n v}=\frac{p_{v}}{P_{n}}$ and $p_{n}=1$ for all values of $n$, then we get a result for $|C, 1|_{k}$ summability. 
Corollary 5. If we take $k=1$ and $a_{n v}=\frac{p_{v}}{P_{n}}$, then we get a result for $\left|\bar{N}, p_{n}\right|$ summability and in this case the condition " $\left(\frac{\varphi_{n} p_{n}}{P_{n}}\right)$ is a nonincreasing sequence" is not needed.

\section{REFERENCES}

1. BARI, N.K.; SteČKIN, S.B. - Best approximations and differential properties of two conjugate functions, (Russian) Trudy Moskov. Mat. Obšč., 5 (1956), 483-522.

2. Bor, H. - On two summability methods, Math. Proc. Cambridge Philos. Soc., 97 (1985), 147-149.

3. Bor, H. - On absolute summability factors, Proc. Amer. Math. Soc., 118 (1993), $71-75$.

4. Flett, T.M. - On an extension of absolute summability and some theorems of Littlewood and Paley, Proc. London Math. Soc., 7 (1957), 113-141.

5. Hardy, G.H. - Divergent Series, Oxford, at the Clarendon Press, 1949.

6. Mazhar, S.M. - Absolute summability factors of infinite series, Kyungpook Math. J., 39 (1999), 67-73.

7. Sulaiman, W.T. - Inclusion theorems for absolute matrix summability methods of an infinite series. IV, Indian J. Pure Appl. Math., 34 (2003), 1547-1557.

Received: 27.III.2012

Revised: 6.VII.2012

Accepted: 13.VII.2012
Department of Mathematics, Erciyes University, 38039, Kayseri, TURKEY seyhan@erciyes.edu.tr keten@erciyes.edu.tr 